\title{
Image segmentation for improved consistency in image-interpretation of opium poppy
}

\author{
Daniel M. Simms, Toby W. Waine, John C. Taylor, Timothy R. Brewer \\ Cranfield University, Bedfordshire, MK43 OAL, United Kingdom
}

\begin{abstract}
The image-interpretation of opium poppy crops from very high resolution satellite imagery forms part of the annual Afghanistan opium surveys conducted by the United Nations Office on Drugs and Crime and the United States Government. We tested the effect of generalisation of field delineations on the final estimates of poppy cultivation using survey data from Helmand province in 2009 and an area frame sampling approach. The sample data was reinterpreted from pan-sharpened IKONOS scenes using two increasing levels of generalisation consistent with observed practice. Samples were also generated from manual labelling of image segmentation and from a digital object classification. Generalisation was found to bias the cultivation estimate between $6.6 \%$ and $13.9 \%$, which is greater than the sample error for the highest level. Object classification of image-segmented samples increased the cultivation estimate by $30.2 \%$ because of systematic labelling error. Manual labelling of imagesegmented samples gave a similar estimate to the original interpretation. The research demonstrates that small changes in poppy interpretation can result in systematic differences in final estimates that are not included within confidence intervals. Segmented parcels were similar to manually digitised fields and could provide increased consistency in field delineation at a reduced cost. The results are significant for Afghanistan's opium monitoring programmes and other surveys where sample data are collected by remote sensing.
\end{abstract}

\section{Introduction}

Annual Statistics on opium cultivation are produced by the United Nations Office on Drugs and Crime/Afghanistan's Ministry of Counter Narcotics (UNODC) and the United States (US) Government to monitor the annual production of illicit opium and evaluate the success of counter narcotics $(\mathrm{CN})$ programmes. They conduct independent surveys based on the extrapolation of area measurements collected at sample locations. The total agricultural area is split into primary sampling units (PSU), from which a statistically representative selection is taken. The cropped area of poppy within each PSU is calculated and the total area of poppy is estimated by multiplying the mean proportion of crop from the sample by the area of agriculture. The surveys differ in their sample proportion, size of PSU and use of stratification.

In both surveys, the area of opium poppy at sample locations is measured by directly digitising crop parcel boundaries from very high resolution (VHR) satellite imagery. Crops are identified by trained interpreters using the standard image-interpretation elements of size, shape, shadow, colour, texture, pattern and association. To maintain consistency, an interpretation key is developed from prior knowledge of the appearance of opium crops in VHR imagery. Keys contain examples of the different crop types and any variation in their appearance with growth stage or management practices.

For accurate area estimates the digitised sample should be a true representation of reality at the sample site. Sources of bias in image interpretation are incorrectly labelled parcels (labelling error) (Gallego, 2006) and imprecision of class definitions relating to what is seen on the imagery e.g. where to place a boundary on a continuum (Foody, 2002). Mapping the true location of parcel boundaries requires a resolution high enough to visualise distinct boundaries between features in the imagery (Goodchild and Hunter, 1997). Other potential sources of bias are the scale of digitisation and the inclusion of 
features within crop polygons due to image resolution or the minimum mapping unit (Carfagna and Gallego, 2005).

The effect of differences in interpretation is the subject of debate between the UNODC and US survey teams as it is difficult to measure and not accounted for in the confidence interval of the final estimate. This article presents the results of research into interpretation bias caused by systematic differences in image-interpretation of poppy. The research questions were: is generalisation in field delineation a significant source of bias in the survey estimate; and can image processing methods improve the consistency of interpretation. This work was part of a wider project for improving cultivation estimates in Afghanistan that took place between 2003-2009, described in Taylor et al. (2010).

\section{Data and methods}

\subsection{Stratified area frame sampling}

A stratified area frame sampling methodology was used as part of the wider investigation into the differences between the UNODC and US surveys. The approach, referred to as GeoTools in some literature, is designed to improve the accuracy of a ratio sample estimate by stratification of the sample frame using satellite imagery (Koeln and Kollasch, 2000). The area of poppy within each stratum $s$ is calculated from $n$ number of samples by

$$
m_{s}=\frac{\sum_{i=1}^{n} m_{i}}{\sum_{i=1}^{n} a_{i}} A_{s},
$$

where $m_{i}$ is the area of the poppy within stratum $s$ in sample $i, a_{i}$ is the total area of sample $i$ in stratum $s$ and $A_{s}$ is the total area of the stratum in the study area. The total area estimate for poppy $(M)$ is the combined estimates for all strata,

$$
M=\sum_{s=1}^{x} m_{s}
$$

The purpose of stratification is to minimise the within-stratum variance compared to the variance between strata by grouping areas that are homogeneous, with low variation in the occurrence of poppy (Cochran, 1977).

The confidence interval of the estimate is calculated by bootstrapping, which uses Monte Carlo simulation to approximate the distribution of $M$ from $N$ repetitions of equation 1 using a random draw of the sample,

$$
M^{*}=M\left(X_{1}^{*}, \ldots, X_{n}^{*}\right)
$$

where $X_{1}^{*}, \ldots, X_{n}^{*}$ is an independant random selection of the original samples with replacement. This results in $N$ calculations of $M^{*}$. The upper and lower confidence intervals are found by ordering the values of $M^{*}$ and taking the value corresponding to the percentile required. For example, $M_{500}^{*}$ and $M_{9500}^{*}$ for the $90 \%$ confidence level for $N=10,000$.

The samples were selected by first defining a $10 \mathrm{~km} \times 10 \mathrm{~km}$ grid coincident with the UNODC's image collection areas, known as blocks, which was sub-divided into $1 \mathrm{~km} \times 1$ $\mathrm{km}$ PSUs. Random $1 \mathrm{~km}$ squares were selected within each block until a $2 \%$ sample was obtained. A map of agricultural production was used to mask out PSUs with less than $20 \%$ of their area in agriculture. 
The research was carried out using a subset of the 2009 Helmand Province area frame sampling dataset, comprising 61 samples interpreted from 14 IKONOS pan-sharpened VHR images (figure 1). The spectral strata were created from a $32 \mathrm{~m}$ resolution multispectral image (red, green, near-infrared wavebands) from the Disaster Monitoring Constellation $(\mathrm{DMC})$.

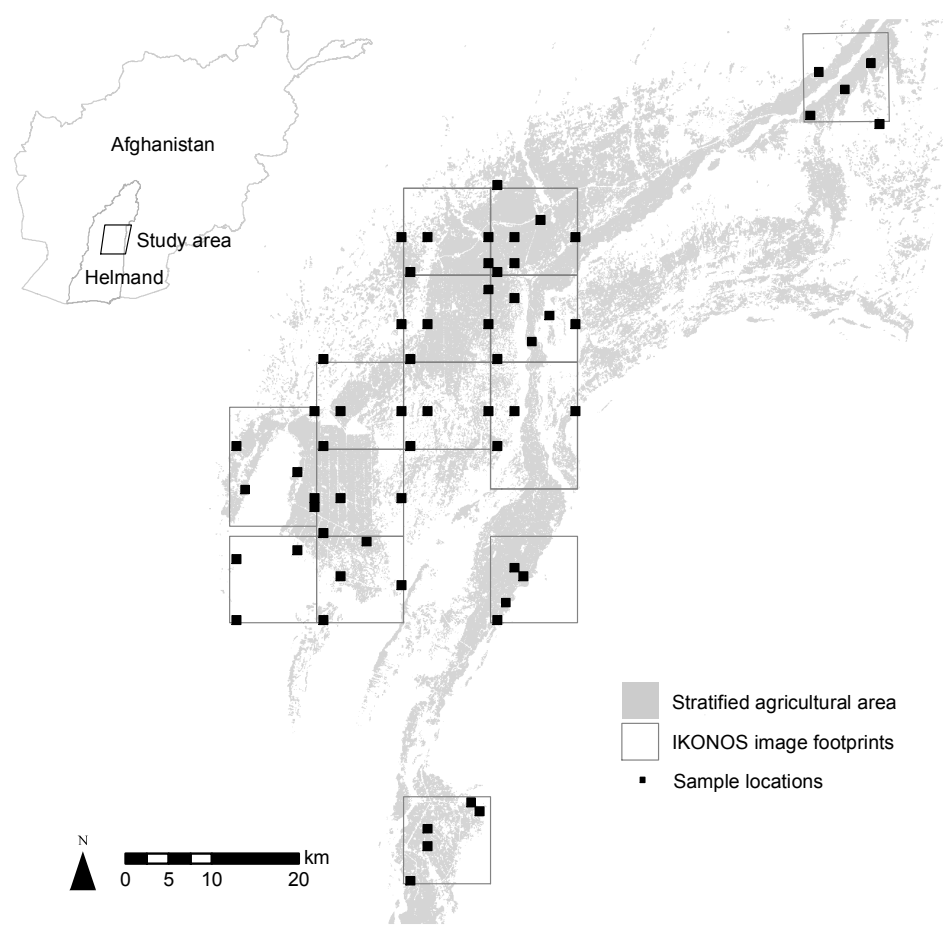

Figure 1: Map showing sample distribution and extent of stratified agricultural area for the central part of Helmand Province, Afghanistan 2009.

\subsection{Image data and processing}

Image acquisition for DMC and IKONOS imagery was timed to coincide with poppy flowering, the optimum growth stage for image-interpretation, using an information system based on time series Normalised Difference Vegetation Index (NDVI) from the Moderate Resolution Spectroradiometer, described in Simms et al. (2014).

The DMC level L1R image, acquired on 25 March 2009 was orthorectified using the bespoke sensor model in Keystone Workstation ${ }^{\circledR}$ software with a controlled image base (CIB) and a $30 \mathrm{~m}$ digital elevation model (DEM), to achieve sub-pixel geometric accuracy. The Iterative Self-Organising Data Analysis Technique (ISODATA) was used to cluster the image pixels into 90 spectral signatures and the image classified using a maximum likelihood discriminant function. The resulting classified pixels were grouped into agriculture and non-agriculture information classes by visual image-interpretation. The nonagricultural classes were then removed and the agricultural mask was manually edited in areas of spectral confusion between natural vegetation and agriculture. The original image was then subset to the area within the agricultural mask and the classification procedure repeated with 30 classes to produce the spectral strata.

The IKONOS geo-bundle images were pan-sharpened using a modified IHS approach to $1 \mathrm{~m}$ resolution (Siddiqui, 2003). The greater spectral range of the IKONOS panchromatic band compared to the combined multispectral bands was found to increase bright- 
ness in the blue band of the pan-sharpened image. This effect was reduced by modifying the panchromatic band to remove part of the near-infrared signal,

$$
\rho_{p}^{*}=\left(1-\frac{\rho_{4}}{\rho_{1}+\rho_{2}+\rho_{3}+\rho_{4}}\right) \rho_{p},
$$

where $\rho_{p}$ is the radiance of the panchromatic band and $\rho_{n}$ is the radiance of multispectral band $n$, before applying the modified IHS algorithm. Each image was then orthoresampled using the vendor supplied Rational Polynomial Camera model refined using control points from the CIB and 30 m DEM (Grodecki and Dial, 2001).

\subsection{Image-interpretation and segmentation}

The frame sampling analysis was conducted for a series of sample sets, each comprising 61 samples, created using 5 different interpretation methods. They were: the original 2009 image interpretations; two levels of increasing generalisation in field boundaries; automatic segmentation with manual classification; and automatic segmentation with object classification.

The original $1 \mathrm{~km}$ samples were image-interpreted from 14 pan-sharpened IKONOS images (table 1). Each image was assessed for crop growth stage and then contrast stretched to optimise the display of the 16 bit data and reduce any distortion during visual display (8 bit) in the software. Sample sites were assigned to trained interpreters who digitised poppy and cereal field parcels using the standard image interpretation method. Poppy crops are distinguished from crops of wheat and alfalfa by visual differences in colour and texture in true-colour and false-colour (near-infrared) VHR composite images. Bare areas of fields and within-field features visible in the imagery were not included within the cropped poppy area.

Table 1: IKONOS image acquisition dates (2009) and poppy growth stage.

\begin{tabular}{rcl}
\hline Date & No. of images & Growth stage \\
\hline 25 March & 7 & Stem elongation \\
3 April & 1 & Flowering \\
8 April & 1 & Flowering \\
11 April & 2 & Flowering \\
25 April & 3 & Capsule \\
\hline
\end{tabular}

Interpretation consistency was cross-checked by assigning $5 \%$ of the samples to multiple interpreters. Systematic differences in interpretation were identified in the overlapping samples and corrected. Consistency in colour representation of crops in false-colour and true-colour composites (stretching) was maintained through supervision and use of auxiliary information on timing from the crop information system. Every sample was cross checked by an experienced interpreter before analysis.

Copies of the original 2009 image-interpreted samples were edited to create two sample sets with increasing levels of generalisation. Samples were first vectorised and polygon boundaries smoothed to improve the cartographic quality. Each sample was then manually re-interpreted using the original VHR imagery. For level 1, paths and singlevehicle width gaps between cropped areas with little or no field margin vegetation were manually removed. Single lines of trees and narrow irrigation channels between polygons were removed by digitising a new boundary along the centre line of the linear feature. Un-cropped areas less than approximately $50 \mathrm{~m}^{2}$ within parcels were merged with the surrounding polygon. Poor quality crops not previously delineated were added to 
the mapped area. Convoluted field boundaries found in polygons where crop density reduced gradually to bare soil were simplified manually.

Level 2 was a further generalisation of the level 1 interpretation. Edits were made to remove paths and single vehicle-width tracks with vegetated margins. Single lines of trees and tracks associated with irrigation channels were removed by digitising a centre line along features. Un-cropped areas greater than $50 \mathrm{~m}^{2}$ within parcels that were interpreted as cultivated were merged with the surrounding polygon. Finally, partial fields with areas of poor or damaged crops were extended to the whole field parcel where there was evidence of an intention to cultivate.

Figure 2 shows examples of level 1 and 2 edits made to sample 39 and sample 36 overlaid on IKONOS near-infrared false colour imagery. At level 1, field polygons for sample 39 include linear features between parcels such as trees, tracks and drainage ditches. Within-field areas of bare soil or poor crop are removed at level 2 and field parcels are extended to boundary edges. In sample 36, patchy areas are extended at level 1 to incorporate more of the poor quality crop and the drainage features that separate parcels. At level 2 the whole block of variable crop becomes a single polygon representing the farmers intention to cultivate.

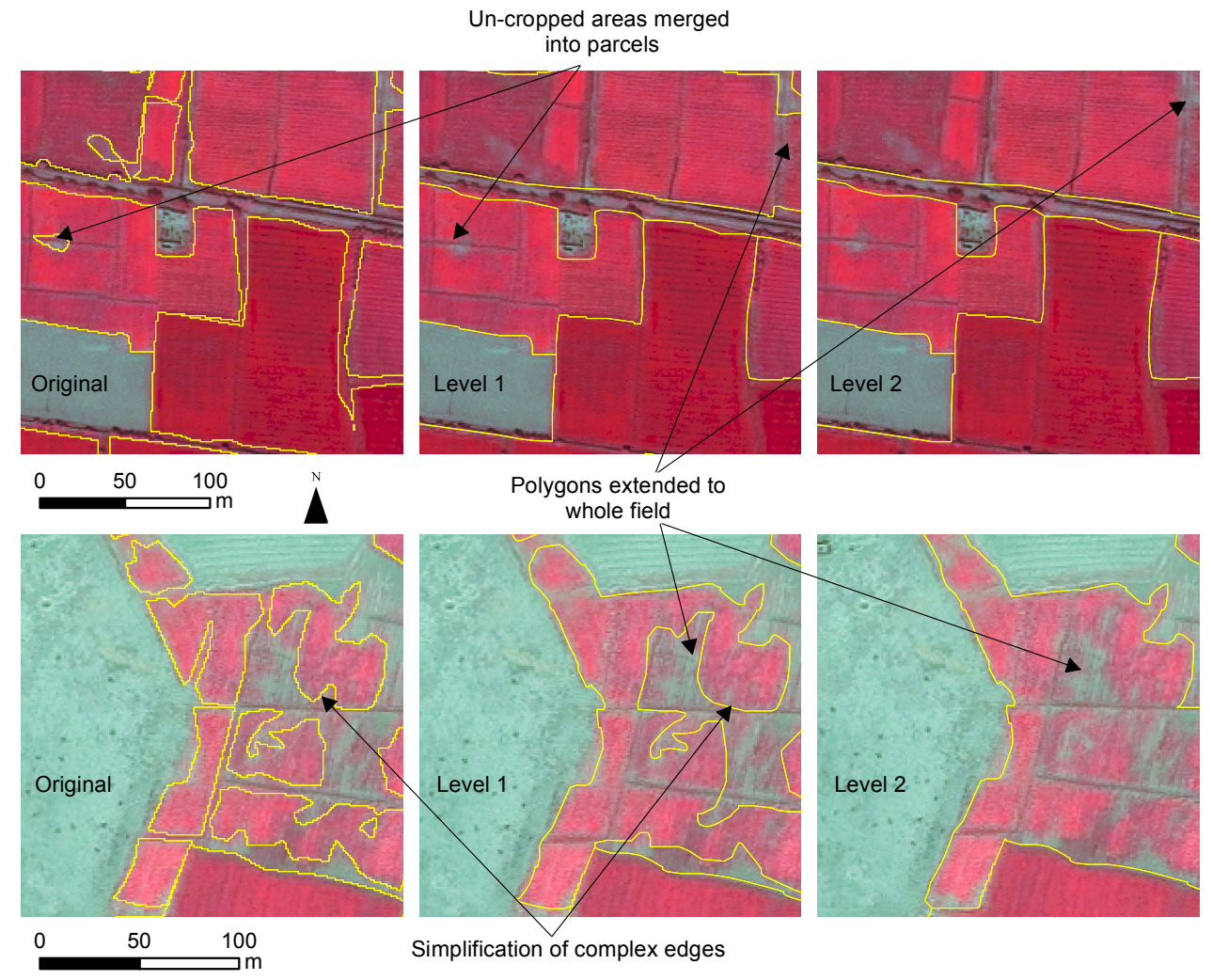

Figure 2: Example of level 1 and 2 edits to sample 39 (top) and 36 (bottom). Boundary delineations overlaid on IKONOS (near-infrared false-colour) imagery. Samples centred on latitude $31.501^{\circ} \mathrm{N}$, longitude $64.187^{\circ} \mathrm{E}$ (39) and latitude $31.503^{\circ} \mathrm{N}$, longitude $64.081^{\circ} \mathrm{E}(36)$.

A third sample set was created to simulate a methodology where images are automatically segmented and the resulting parcels classified manually by image-interpretation. Automated segmentation was performed for each IKONOS image used for interpretation using eCognition ${ }^{\circledR}$ software. The software uses a bottom-up region merging technique to group homogeneous pixels into objects of similar size and scale based on a scaling fac- 
tor (Benz et al., 2004). A scaling factor of 80 was determined by systematic testing and found to be suitable for the segmentation of opium and cereal parcels from the $1 \mathrm{~m}$ resolution IKONOS images. A single level segmentation was then run on each image using the same scaling factor and homogeneity criteria of 0.1 for shape factor and 0.5 for compactness (Baatz et al., 2004). Segmented polygons were then intersected with the original 2009 samples and their classes assigned by selecting the majority class from the original interpretation for each polygon.

The final sample set was created by conducting an object based nearest neighbour classification within the software for the segmented polygons in each IKONOS scene. The classifier was trained using fields of poppy and cereal selected in areas away from the sample squares. Classified samples were then extracted at coincident locations to the 2009 sample. A small number of anomalies were found in the samples caused by unclassified areas in the object classification. These areas were masked out of all 5 sample sets.

Each of the 61 samples in each set were rasterised at a grid resolution of $1 \mathrm{~m}$ to match the format of the original 2009 samples. The different levels of generalisation and automatic methods were then compared by running 5 separate stratified area frame sampling analyses to estimate the total area of poppy using the same 30 spectral strata.

\section{Results}

Table 2 shows the poppy area estimates for the 5 interpretation methods. The estimates range from 39534 to 51463 ha and have a similar lower (about 8\%) and upper (about 10\%) confidence interval (90\%). Generalisation of interpretation increases the poppy estimate by $6.6 \%$ for level 1 and by $13.9 \%$ for level 2 . The increase in the poppy estimate for the level 2 interpretations is greater than the upper confidence interval using the original sample $(10.4 \%)$. Automatic segmentation of the field parcels with manual class assignment increased the estimate by $2.4 \%$, the smallest difference of all methods from the original sample estimate. The object classification increases the estimate by $30.2 \%$ from the original estimate.

Table 2: Poppy area estimates and 90\% confidence intervals for different levels of generalisation and automatic segmentation using 61 samples in the Helmand trial area, with percentage difference from the original (* outside confidence interval).

\begin{tabular}{lrrrr}
\hline Method & Area (ha) & Upper (\%) & Lower (\%) & Diff. (\%) \\
\hline Original & 39534 & 8.5 & 10.4 & \\
Level1 & 42145 & 8.1 & 10.5 & 6.6 \\
Level2 & 45031 & 8.3 & 10.6 & $* 13.9$ \\
Segments manual & 40488 & 8.4 & 11.0 & 2.4 \\
Segments trained & 51463 & 7.4 & 9.0 & $* 30.2$ \\
\hline
\end{tabular}

Figure 3 shows the individual poppy proportions for each interpretation method plotted against the original samples. For the level 1 and level 2 (figure 3(a) and 3(b)) the generalisation in the interpretation creates a positive bias in sample proportion that increases with the proportion of poppy in the sample. In figure 3(c), the proportion of poppy in automatically segmented samples is similar to the original interpretation proportions. The object based classification of the samples (figure 3(d)) shows a positive bias towards poppy and a reduction in the coefficient of determination $\left(R^{2}\right)$ to 0.84 from $>0.99$ for the other methods.

Figure 4 shows a visual comparison of part of sample 67 for the different automatic methods with manual interpretation. The results of the segmentation overlaid on the 


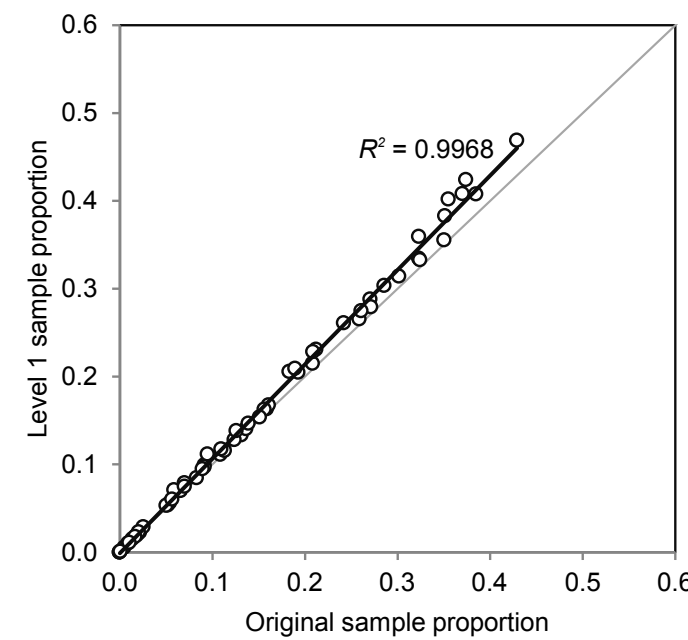

(a)

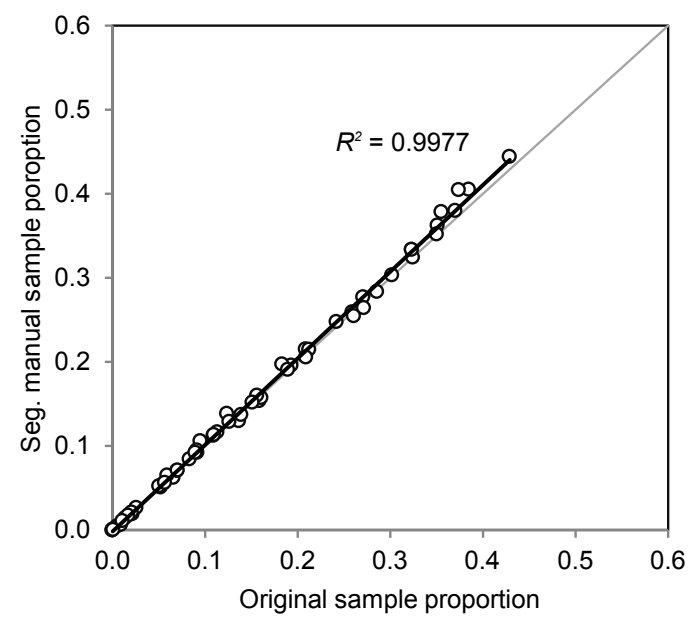

(c)

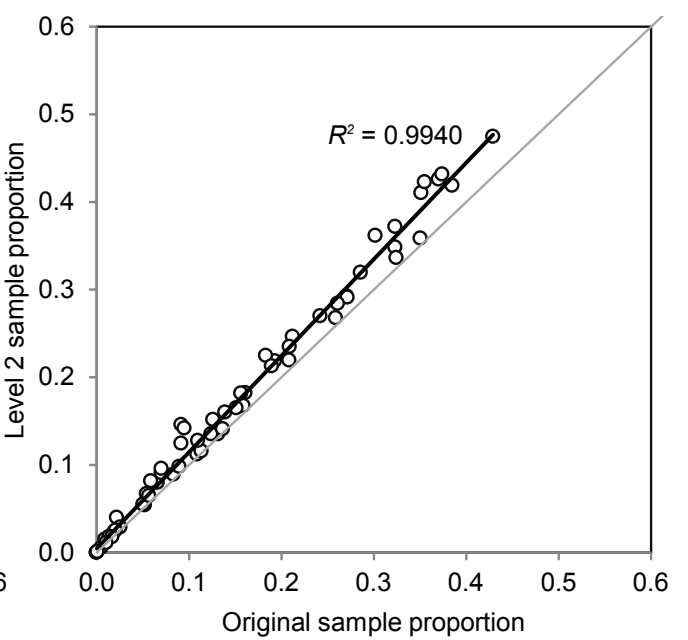

(b)

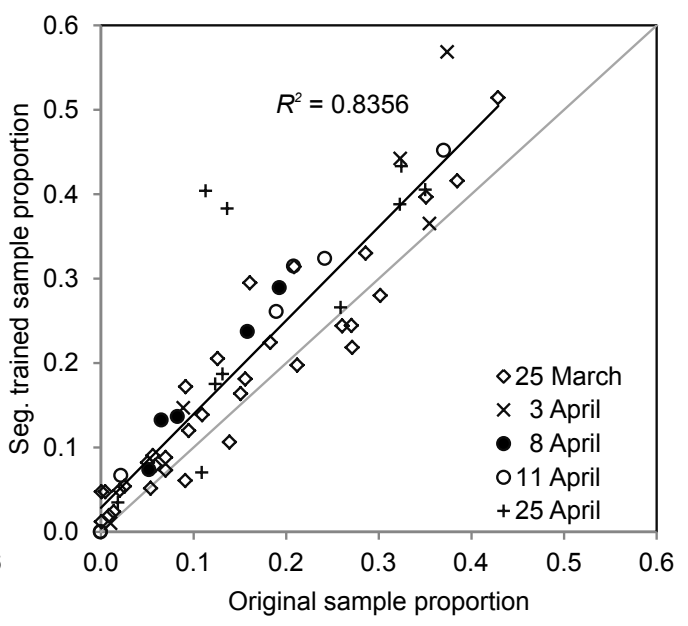

(d)

Figure 3: Linear regression of (a) level 1 and (b) level 2 generalisations, (c) manually assigned and (d) digitally classified segments (seg.) with original poppy proportions for all 61 samples.

IKONOS image (figure 4(a)) show accurate delineation of field parcels, within-field bare patches and tree-lined boundaries between parcels. These objects match the general shape of manually interpreted field parcels (figure 4(b)) and the manual classification of the objects (figure 4(c)) shows good agreement with the original interpretation. However, differences can be seen in the complexity of the parcel edges and in cases where single objects from the segmentation are split in the image-interpretation. Heterogeneous areas in the imagery, where multiple linear features intersect with small parcels, are incorrectly labelled as the segmented objects cover multiple classes.

In the object-classified sample (figure 4(c)) there are errors in the classification of field parcels and boundary features. A confusion matrix of the classified and original imageinterpreted samples is shown in table 3. Assuming the visual interpretation as the reference data, the user accuracy of the object classification of poppy is $59 \%$ with a higher commission error compared to the omission error. This shows a bias towards the classification of poppy that increases the overall estimate. 


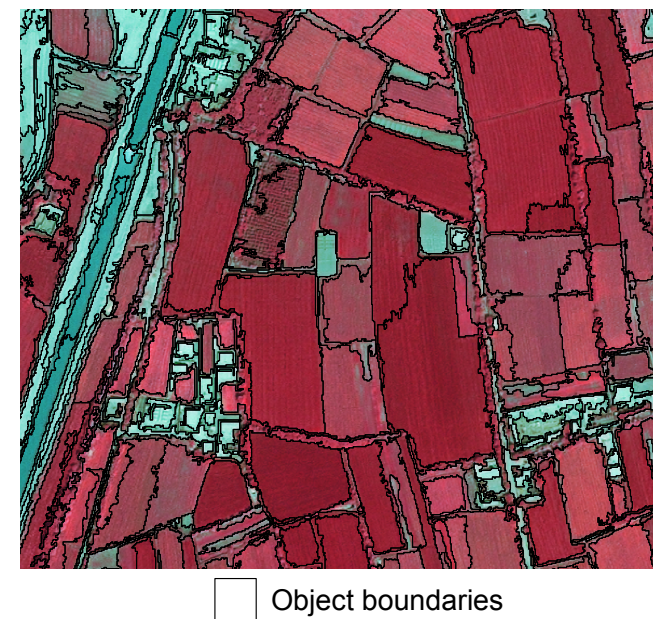

(a) Segmentation boundaries

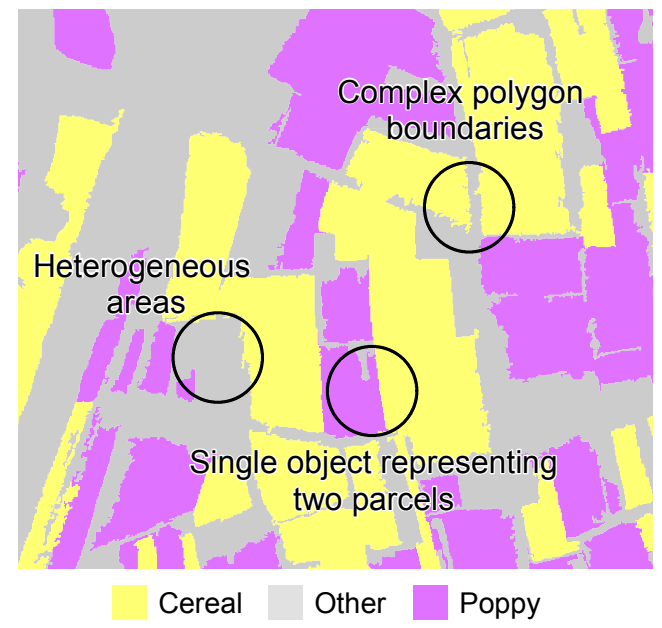

(c) Manually assigned object classes

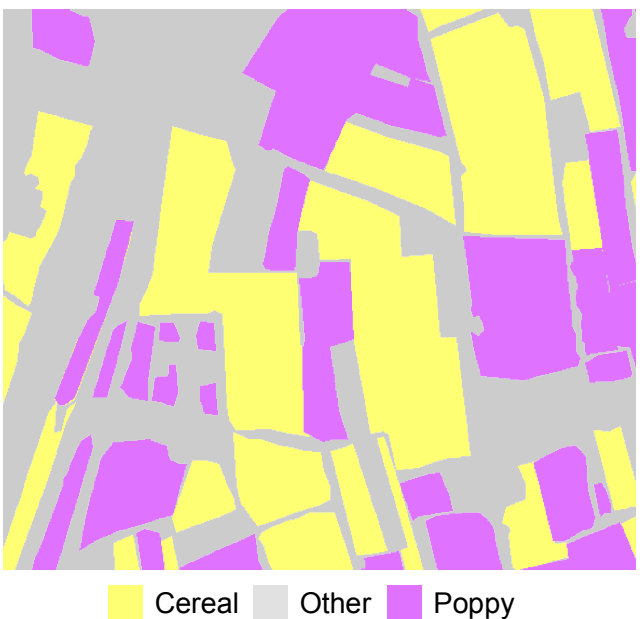

(b) Original interpretation

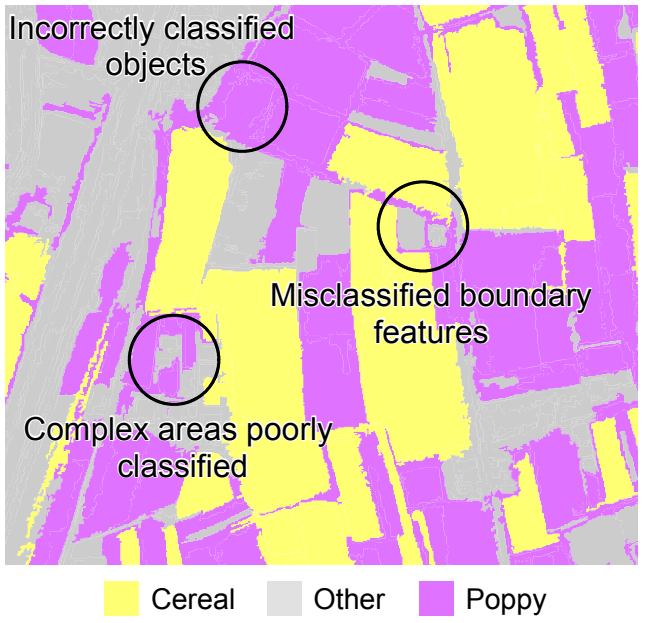

(d) Object classification

Figure 4: Example of manual interpretation and classification of automatically segmented field parcels from pan-sharpened IKONOS imagery.

\section{Discussion}

We have tested the two sources of sample interpretation error that could bias the final area estimate. They are the delineation of the parcel boundaries and the misclassification of crop types within parcels (labelling error). As expected, the results show a positive bias in the sample estimate from generalising the sample interpretations. What is significant is the magnitude of the bias: for the higher level of generalisation it is greater than the sample error estimated from the bootstrap (13.9\% vs $10.6 \%)$.

There are several factors in manual image-interpretation that can lead to the levels of generalisation investigated. The first is a tendency for interpreters to digitise fewer vertices in parcel boundaries to speed up the interpretation of individual samples. This is particularly the case when delineating large blocks of contiguous fields that contain the same crop type. Within-field features such as irrigation ditches and linear features between fields are more likely to be included within parcel boundaries to improve the interpreters' productivity. 
Table 3: Confusion matrix of 61 object classified and image-interpreted samples (as proportions), rasterised to $1 \mathrm{~m}$.

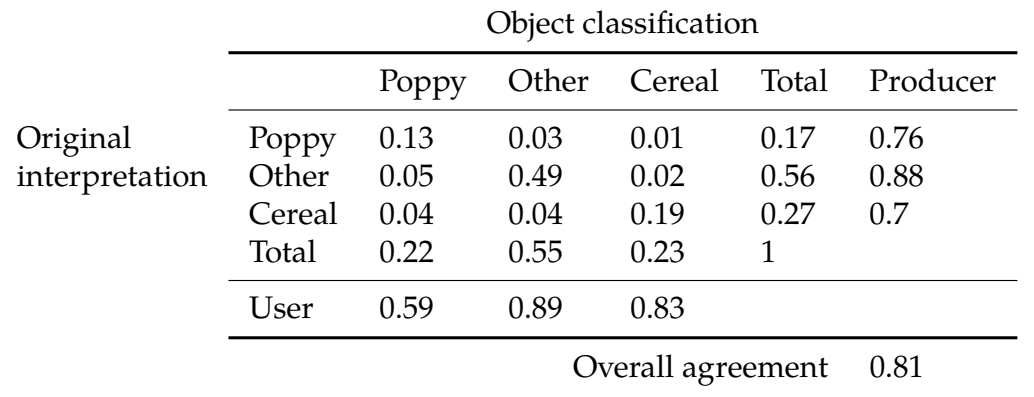

The second factor is the definition of a field parcel in the interpretation key. If the interpreter is tasked with identifying the farmers' intention to cultivate a certain crop, the field delineation will include within-field bare patches and poor crops by design. In crops with a uniform canopy this approach will produce similar results to interpretations delineating the actual visible crop area. However, in areas of marginal agriculture or in years with poor crop establishment this will greatly affect the proportions of the target crop within samples and the resulting area estimates.

The third factor is the scale of digitised crop areas and is related to the resolution of imagery. Interpreters using aerial digital photography could be able to accurately delineate areas of thin crops and within-field features that are not visible in lower resolution satellite imagery. In marginal areas or in crops with poor establishment this will create systematic differences in interpretation related to the appearance of thin parcels of poppy and the size of within-field features in the imagery. If the area of these small features makes a significant contribution to the cropped area of poppy the sample interpretations will become unreliable.

Finally appearance of crops in imagery changes according to their growth stage. Errors could be introduced by interpretation of underdeveloped crop canopies from images collected early in the growing season. In the example from 2008 shown in figure 5, background soil is visible through the canopy within poppy fields on 28 March (figure 5(a)) that is subsequently covered by the time of the second image on 27 April (figure 5(b)). The early interpretation (yellow lines) excludes parts of the field at the earlier date that are included in the later interpretation. In 2008, poor crop establishment due to cold spring weather caused visible differences in the crop canopy at the stem elongation growth stage. In a normal year the canopy would be expected to be fully developed at this growth stage and within canopy bare patches digitised out during interpretation as being un-cropped. Figure 6 shows another area from Helmand Province in 2008 where the early damage to the crop has resulted in bare patches (figure 6(a)) that are still visible in the later image (figure 6(b)). Inclusion of these areas within the samples will lead to an over estimation of the cropped area of poppy.

The effect of these factors will vary between groups of interpreters according to their specific training, the interpretation key and the imagery source; and also between interpreters within the same group. Methods to maintain accuracy and consistency across samples are standard practice for surveys that rely on image-interpretation and include comparisons of sample interpretations between individuals; review of samples by more experienced interpreters; and multiple-pairs-of-eyes, where teams of interpreters consider marginal cases together.

Controls to limit the level of generalisation require more resources as the area of the 

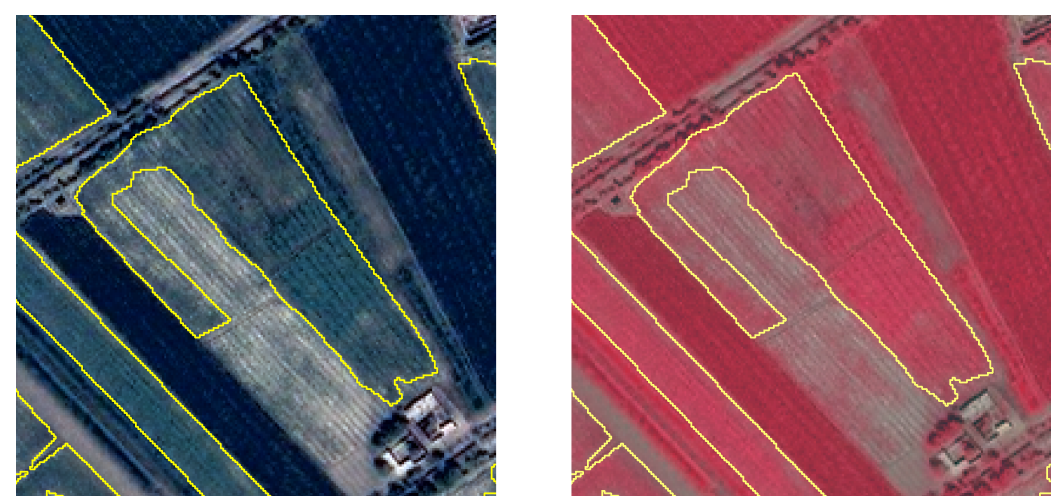

(a) 28 March 2008
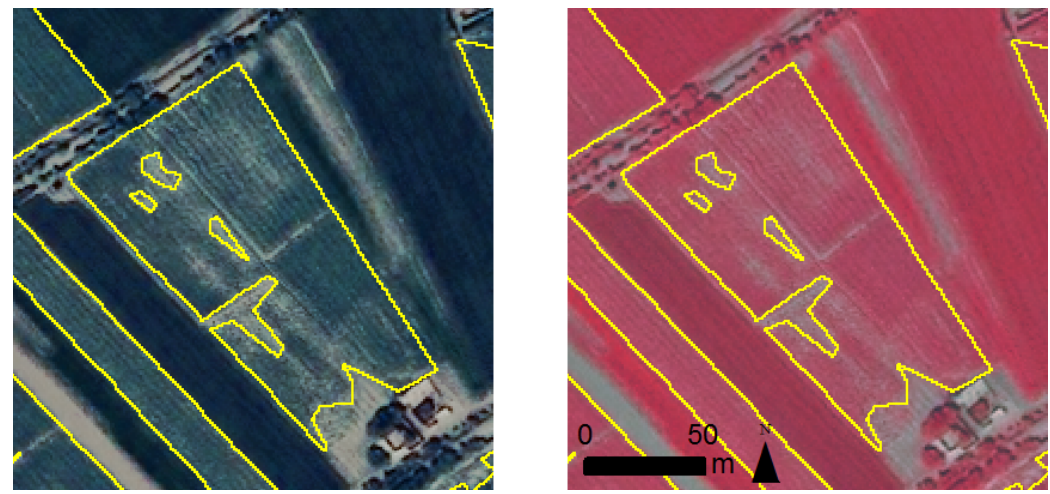

(b) 27 April 2008

Figure 5: True-colour and false-colour subsets of pan-sharpened IKONOS images for two dates showing area of poppy cultivation in Helmand Province with poor crop establishment. Poppy fields delineated in yellow, subsets centred at latitude $31.687^{\circ} \mathrm{N}$, longitude $64.284^{\circ} \mathrm{E}$.

sample increases. Smaller samples allow for shorter, more focused analysis and are easier to cross reference to maintain consistency between individual interpreters. Conversely, large samples (e.g. an entire VHR image) that include hundreds of fields are more likely to contain field boundary generalisations and omissions of within-field features, especially in areas dominated by the crop of interest. They are also more difficult to quality check and cross reference. The effect of interpreter generalisation is compounded in larger samples with poor crop establishment; where accurate digitisation of complex field parcels is a significant increase in the work load of the interpreter.

Segmentation and object classification were investigated for potential improvements to the consistency and speed of sample interpretation. Image segmentation produced similar results to the manual delineation of field parcels by interpreters. In the case of eCognition, the manual steps of the segmentation are limited to the selection of suitable homogeneity criteria, which were found to be constant across image scenes in this study. Once this is done whole images can be segmented in minutes and the work of the interpreter is focused on the labelling of field parcels with some minor editing of boundary errors in complex areas. This speeds up interpretation and prevents generalisation that might arise from interpretation of contiguous blocks of the same crop and complex field boundaries. Further research into optimising the segmentation of poppy crops and the effect of growth stage and image resolution on the accuracy of segmented field parcels is necessary to support its use in operational surveys.

Totally automatic methods limit the effort of image-interpretation to a subset of rep- 

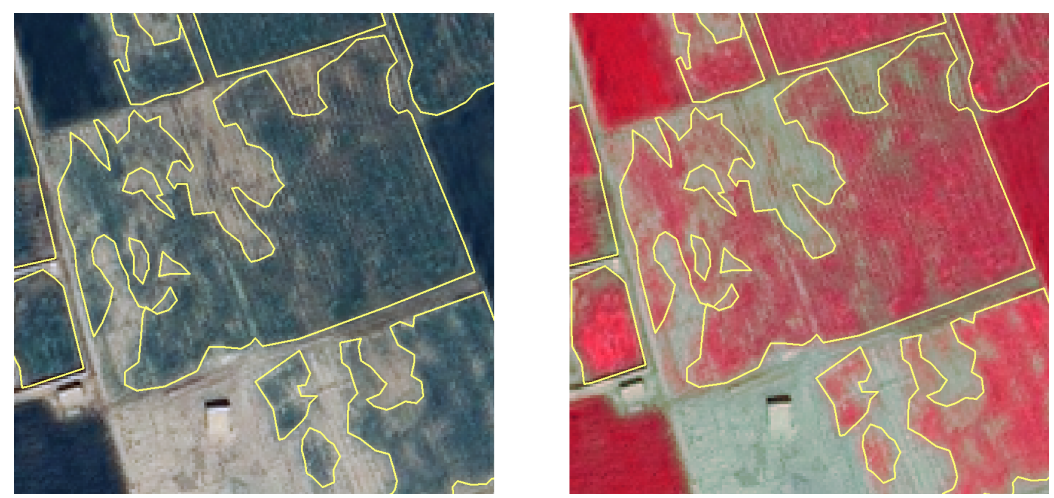

(a) 27 March 2008
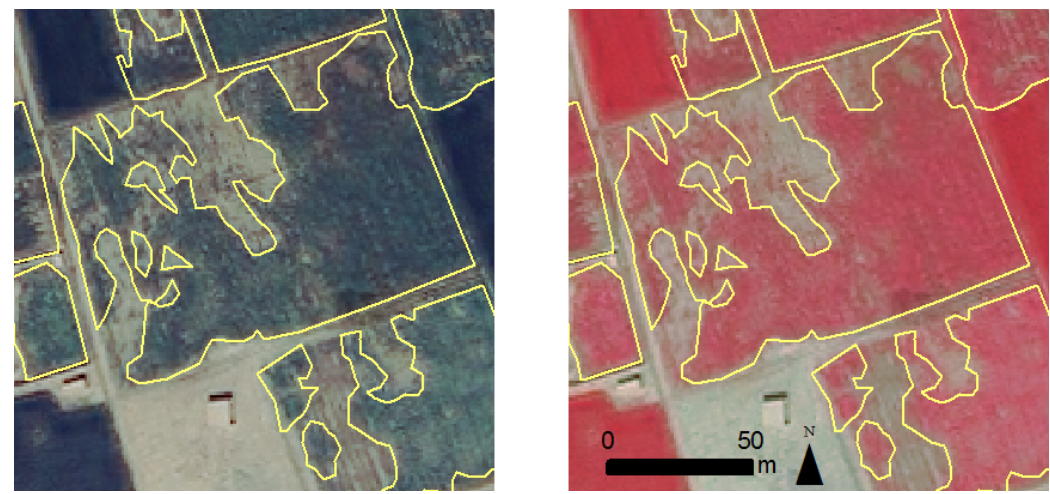

(b) 27 April 2008

Figure 6: True-colour and false-colour subsets of pan-sharpened IKONOS images for two dates showing area of poppy crops damaged by cold weather that did not recover, Helmand Province. Poppy fields delineated in yellow, subsets centred at latitude $31.739^{\circ} \mathrm{N}$, longitude $64.332^{\circ} \mathrm{E}$.

resentative fields for training and evaluating the classifier. The object classification was found to be unsuitable for automatic interpretation as the systematic error in the classified samples biases the final cultivation figure (30.4\% increase in poppy area for this study). These results highlight the importance of systematic bias correction for obtaining accurate area estimates from image classifications, as discussed for pixel based classifiers by Gallego (2004).

Provided the quality of interpretation can be controlled, survey interpretations can be consistent within survey teams across growing seasons for comparisons of inter-annual estimates. However, differences in interpretation keys - relating to the imagery and the definition of the interpretation classes - are likely to be a source of disagreement between estimates from independent surveys. Within the context of the annual opium surveys in Afghanistan, consistency between the UNODC and US estimates was greatly improved from 2005 through sharing of interpretations at overlapping sample sites (Taylor et al., 2010). Differences in crop classification were reconciled and systematic differences in the interpretation approach were identified, leading to harmonisation of cultivation estimates without affecting the independence of the surveys.

\section{Conclusions}

Generalisation in sample interpretation results in systematic differences in final estimates of poppy that are not accounted for in the confidence interval of the final estimate. Es- 
timates were $6.6 \%$ and $13.9 \%$ higher for generalised samples, which is greater than the confidence interval for the higher level of generalisation. These results show that disagreement in annual estimates between Afghanistan's monitoring programmes can result from systematic differences in class definitions and interpretation keys for poppy.

Image segmentation produced similar parcel boundaries to manual digitising. The manual labelling of image segments shows potential for increasing the speed of interpretation while maintaining a consistent delineation of field parcels. Further research is required to optimise the segmentation of images collected at different crop growth stages and to investigate the effect of VHR image resolution before operational use. Object based classification of VHR imagery was found to be unsuitable for samples production because of low labelling accuracy.

This work highlights the requirement for controls to maintain the consistency of interpretation. Suitable class definitions and keys relating to the features visible in VHR imagery are essential to reduce differences between individuals and teams of interpreters. We recommended splitting larger samples to allow for shorter, more focused analysis and improved quality control.

The results are significant for surveys that use visual interpretation of remotely sensed data. Imagery must be of the appropriate resolution and class definitions applicable to observable differences in the imagery to capture a true representation of reality and avoid bias.

\section{Acknowledgements}

The authors would like to thank the UK government for sponsoring the research.

\section{References}

BaAtz, M., Benz, U., Dehghani, S., Heynen, M., Holtje, A., Hofmann, P., Lingenfelder, I., Mimler, M., Sohlbach, M. and Weber, M., 2004, eCognition Professional User Guide 4.

Benz, U.C., Hofmann, P., Willhauck, G., Lingenfelder, I. and Heynen, M., 2004, Multiresolution, object-oriented fuzzy analysis of remote sensing data for GIS-ready information. ISPRS Journal of Photogrammetry and Remote Sensing, 58(3-4), pp. 239-258. URL http://dx.doi.org/10.1016/j.isprsjprs.2003.10.002

Carfagna, E. and Gallego, F.J., 2005, Using Remote Sensing for Agricultural Statistics. International Statistical Review, 73(3), pp. 389-404. URL http://dx.doi.org/10.1111/ j.1751-5823.2005.tb00155.x

Cochran, W.G., 1977, Sampling Techniques, 3rd ed., John Wiley \& Sons, New York.

Foody, G.M., 2002, Status of land cover classification accuracy assessment. Remote Sensing of Environment, 80(1), pp. 185-201. URL http://dx.doi.org/10.1016/ S0034-4257 (01) 00295-4

GALLEGO, F.J., 2004, Remote sensing and land cover area estimation. International Journal of Remote Sensing, 25(15), pp. 3019-3047. URL http://www . informaworld.com/10 . 1080/ 01431160310001619607

Gallego, F.J., 2006, Review of the main remote sensing methods for crop area estimates, in ISPRS WG VIII/10 Workshop 2006, vol. Stresa, It. 
GoodchiLd, M.F. and HunTer, G.J., 1997, A simple positional accuracy measure for linear features. International Journal of Geographical Information Science, 11(3), pp. 299-306. URL http://dx.doi.org/10.1080/136588197242419

Grodecki, J. and Dial, G., 2001, IKONOS Geometric Accuracy, in Proceedings of Joint Workshop of ISPRS Working Groups I/2, I/5 and IV/7 on High Resolution Mapping from Space 2001, pp. 19-21.

Koeln, G.T. and Kollasch, R.P., 2000, Crop area assessments using low, moderate, and high resolution imagery: a Geotools approach, in ASPRS Annual conference, Washington, D.C.

Siddiqui, Y., 2003, The modified IHS method for fusing satellite imagery, in ASPRS Annual conference, Anchorage, Alaska.

Simms, D.M., Waine, T.W., TAYlOR, J.C. and JuniPeR, G.R., 2014, The application of timeseries MODIS NDVI profiles for the acquisition of crop information across Afghanistan. International Journal of Remote Sensing, 35(16), pp. 6234-6254. URL http: //dx . doi . org/ $10.1080 / 01431161.2014 .951099$

Taylor, J.C., Waine, T.W., Juniper, G.R., Simms, D.M. and Brewer, T.R., 2010, Survey and monitoring of opium poppy and wheat in Afghanistan: 2003-2009. Remote Sensing Letters, 1(3), pp. 179-185. URL http: //www . informaworld.com/10 .1080/ 01431161003713028 